\title{
Flame curvature distribution in high pressure turbulent Bunsen premixed flames
}

M. Klein*a , H. Nachtigal*, M. Hansinger*, M. Pfitzner* and N. Chakraborty**

*Department of Aerospace Engineering, Universität der Bundeswehr München, Neubiberg, 85577, Germany

** School of Mechanical \& Systems Engineering, Newcastle University, Newcastle-UponTyne, NE1 7RU, UK

*a Corresponding author

Tel.: +49-89-60042122

Fax: +49-89-60042135

E-mail: Markus.Klein@unibw.de 


\begin{abstract}
The flame curvature statistics of turbulent premixed Bunsen flames have been analysed in this paper using a Direct Numerical Simulation (DNS) database of turbulent Bunsen flames at ambient and elevated pressures. In order to be able to perform a large parametric study in terms of pressure, heat release parameter, turbulence conditions and nozzle diameter, a single step Arrhenius type irreversible chemistry has been used for the purpose of computational economy, where thermo-chemical parameters are adjusted to match the behavior of stoichiometric methane-air flames. This analysis focuses on the characterization of the local flame geometry in response to turbulence and hydro-dynamic instability. The shape of the flame front is found to be consistent with existing experimental data. Although the Darrieus Landau instability promotes cusp formation, a qualitatively similar flame morphology can be observed for hydrodynamically stable flames. A criterion has been suggested for the curvature PDF to become negatively skewed.
\end{abstract}

Keywords: Direct Numerical Simulation, Turbulent premixed combustion, Flame curvature, Bunsen flame 


\section{INTRODUCTION}

In many technical applications, such as gas turbine combustion chambers or supercharged piston engines, combustion takes place at elevated pressure. Fundamental understanding and reliable modelling of turbulent premixed flames at high pressures are therefore of considerable practical importance [1]. However, despite considerable efforts during the last decades there are crucial gaps in understanding regarding the following aspects:

(i) Large Eddy Simulation (LES) provides a promising compromise between Direct Numerical Simulations (DNS), which resolves all length and time scales of fluid turbulence without any recourse to physical approximations, and the Reynolds averaged Navier Stokes approach (RANS) where all turbulent scales have to be modelled. However, in existing LES subgrid combustion models the effect of pressure has rarely been taken into account (e.g. [2] and references therein) and the existing models are often insufficiently validated regarding turbulent high-pressure combustion. The occurrence of hydro-dynamic instabilities, which can lead to a stronger flame wrinkling, is also often ignored [3].

(ii) In terms of physical understanding of a turbulent premixed flame the turbulent flame speed is a quantity of paramount importance. However, a universal scaling law for the turbulent flame speed is yet to be reported [4] and is still a subject of current research [4,5,6]. Upon onset of hydro-dynamic Darrieus-Landau (DL) instability, the well-known quadratic scaling (i.e. $\left(\mathrm{S}_{\mathrm{T}}-\right.$ $\left.\mathrm{S}_{\mathrm{L}}\right) / \mathrm{S}_{\mathrm{L}} \propto \mathrm{u}^{\prime 2} / \mathrm{S}_{\mathrm{L}}^{2}$ where $\mathrm{S}_{\mathrm{T}}$ denotes the turbulent flame speed, $\mathrm{S}_{\mathrm{L}}$ is the laminar speed of the flame front and $\mathrm{u}^{\prime}$ is the turbulent root-mean-square velocity fluctuation) is replaced by subunity scaling exponents of $\mathrm{u}^{\prime} / \mathrm{S}_{\mathrm{L}}$ according to the findings of Creta and Matalon [4]. Hence, in terms of a more general scaling law [13] of the form $\left(S_{T}-S_{L}\right) / S_{L} \propto\left(u^{\prime} / S_{L}\right)^{n}$, it could be expected that the occurrence of DL instability acts to reduce the scaling exponent $\mathrm{n}$ [6]. Creta et al. [7] show that $S_{\mathrm{T}}$ exhibits an enhancement in the presence of DL instability. Akkerman and Bychkov [5] indicate that in many experiments on turbulent flames the DL instability is 
more important than the turbulence in determining $S_{\mathrm{T}}$. According to Creta and Matalon [4] $\mathrm{S}_{\mathrm{T}}$ can exceed the laminar flame speed by nearly $50 \%$ even at moderate turbulence intensities whereas the scaling exponent is considerably reduced in the range 0.6-0.9. The DL instability of a thermo-diffusively neutral flame (i.e. with global Lewis number of unity) is most effective in an intermediate range of length scales: very small scales are stabilized by the influence of diffusion [4]. Furthermore there is a cutoff wavelength $\lambda_{c}$ such that the flame becomes DL unstable only for wavelength larger than $\lambda_{c}$. This behaviour is illustrated in Fig. 1 using the dispersion relation proposed by Matalon and Matkowsky [9].

(a)

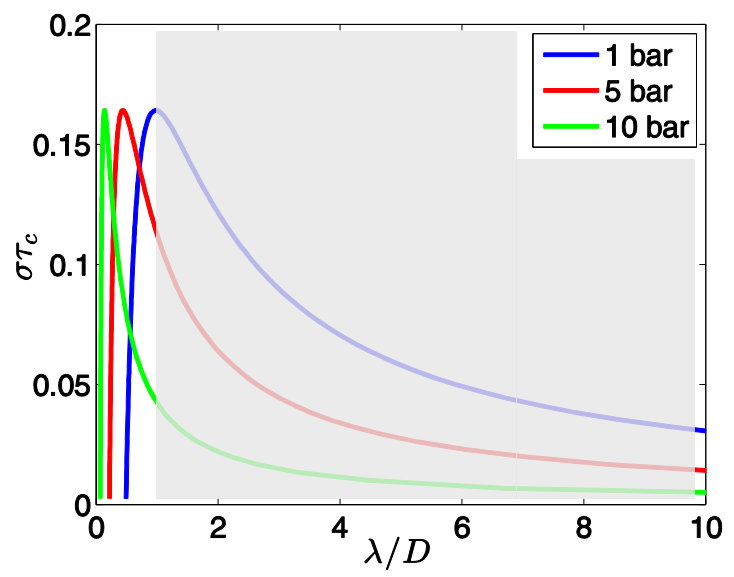

(b)

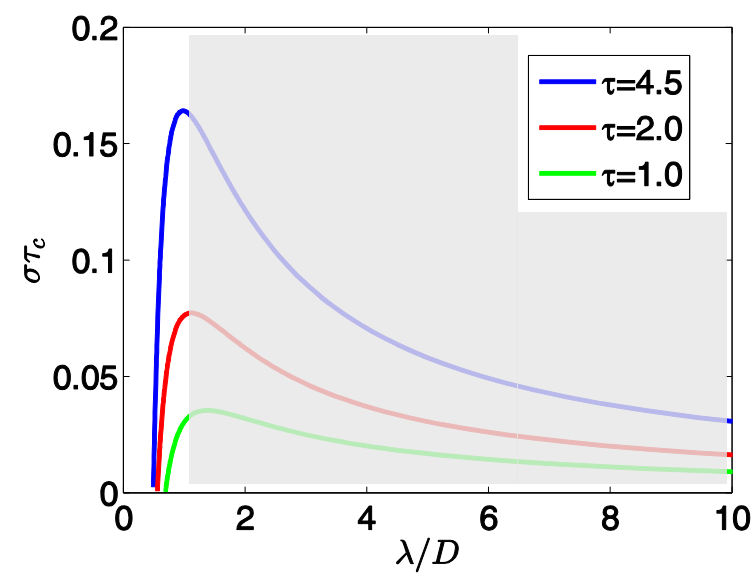

Figure 1. Dispersion relation according to Matalon and Matkowsky [9]. Growth rate $\sigma$ normalised with chemical time scale $\tau_{c}$ versus wavelength divided by nozzle diameter $D$ for (a) Three different pressure levels (b) Three different heat release parameters. Wavelength larger the nozzle diameter are greyed since they are unlikely to occur.

According to the closed form relation for $\lambda_{c}$ derived in [8], which accounts for curvature and hydro-dynamic strain rate in the flame speed stretch relation, the cutoff wavelength is proportional to the Markstein length which itself is proportional to the Zel'dovich flame thickness $\delta_{\mathrm{L}}$ (i.e. the ratio of turbulent diffusivity $\mathrm{D}_{\text {th }}$ to unstreched laminar flame speed $\mathrm{S}_{\mathrm{L}}$ ) [8]. Since $\delta_{\mathrm{L}}$ decreases with increasing pressure the DL induced instability becomes more relevant with increasing pressure. Explicit dependence of the instability on thermo-chemical parameters has been analyzed in $[9,10]$. A comprehensive overview of different approaches of 
modelling and understanding flame instabilities is summarized in Lipatnikov and Chomiak [11].

In order to model and understand the turbulent flame propagation it is therefore important to decide if a flame is hydro-dynamically unstable or not. Creta et al. [7] find that the skewness of the flame curvature probability density function (PDF) is an unambiguous marker for the presence of DL instability. However, the occurrence of rounded leading edges toward the unburned mixture and sharp and narrow cusps can be explained without referring to DL instability. In fact, the phenomena of cusp formation towards the burned gas and the skewed curvature PDF obtained by Shepherd and Ashurst [12] assuming a zero-thickness constantdensity (i.e. absence of heat release and hence DL instability) premixed flame model, compare well with experimental data. The cusp formation can be explained by referring to the Huygens principle since the flame surface propagates normal to itself. Fig. 2 illustrates the simplest situation of flame propagation in a quiescent flow field assuming a constant flame propagation speed of unity, by solving the following simplified form of the $G$-equation [13]: $\partial G / \partial t=|\nabla G|$.

(a)

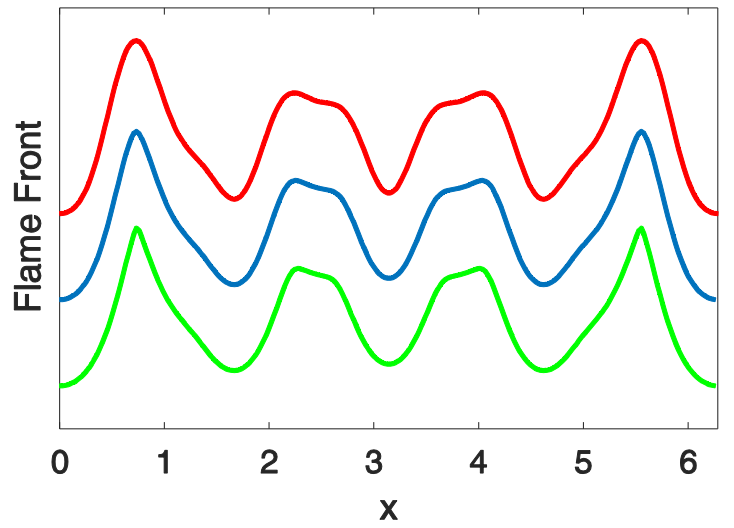

(b)

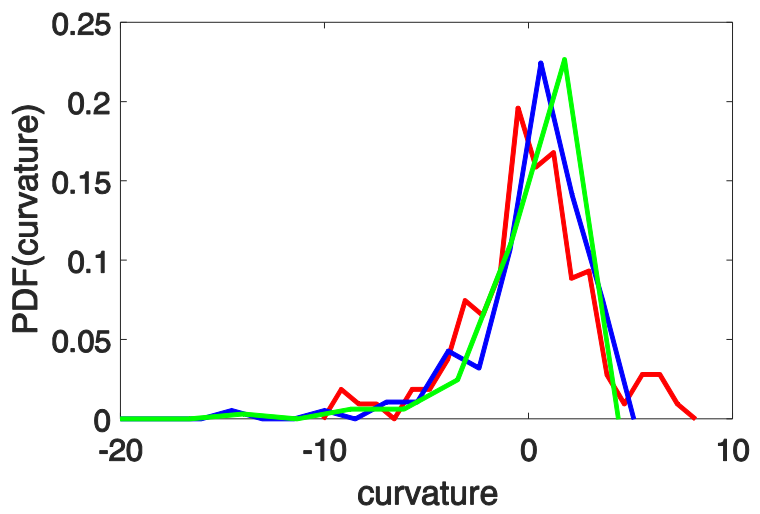

Figure 2. Illustration of Huygens principle: (a) Evolution and propagation of an initial flame surface composed of a few sinusoidal components in a quiescent medium. Flame moves from top to bottom. Red, blue and green line correspond to initial, intermediate and final time step. (b) Corresponding curvature PDFs.

It is worth mentioning that due to the stretch dependence of flame speed, cusps are to some extent smoothed out compared to the so called Landau limit (i.e. independence of $\mathrm{S}_{\mathrm{L}}$ in response 
to stretch) [14]. The asymmetric influence of the laminar burning velocity on the shape of the flame front is also mentioned in [15]. Certainly a hydro-dynamic instability will increase the growth rates of certain wavelength and consequently it will reduce the cusp formation time compared to the corresponding time in absence of turbulence, as shown in [14] and also relative to the turbulent eddy turnover time. Nevertheless, it remains an open question if the skewness of the curvature PDF can be considered equivalent to the presence of hydro-dynamic instability.

It is known that an enhancement of $S_{T}$ due to hydro-dynamic instability decreases with increasing turbulence intensity. However, it is not clear if this will result in a single scaling law beyond a critical threshold, as argued in [16], or not. Finally, accurate modelling of high pressure flames including flame instabilities in the context of Large Eddy Simulation remains an open issue. In order to address some of the aforementioned problems a DNS database of turbulent premixed Bunsen flames at elevated pressures has been generated and analysed. The main focus of this work will be on the analysis of curvature statistics which are important for modelling displacement speed and have been suggested as a marker for DL instability [7]. Future work will address the scaling of the turbulent flame propagation and the modelling of instabilities in the context of LES.

The rest of the paper is organized as follows: The details related to the DNS database will be explained in the next section. This will be followed by the analysis of the flame geometry. Finally, conclusions will be drawn.

\section{DNS DATABASE}

A DNS database of turbulent premixed Bunsen flames consisting of 15 flames denoted henceforth as cases A to V (it has to be noted, that a few cases are not discussed in the context of this work) with global Lewis number $L e=1.0$ has been considered for the present analysis. Because this work reports a computationally expensive, large parametric analysis, a single step 
Arrhenius type irreversible chemistry has been used. The Reynolds number $\operatorname{Re}=\mathrm{U}_{\mathrm{B}} \mathrm{D} / \nu_{\mathrm{u}}$ based on the bulk inlet velocity $\mathrm{U}_{\mathrm{B}}$, nozzle diameter $\mathrm{D}$, and the kinematic viscosity in the unburned gas $v_{\mathrm{u}}$, the turbulent Reynolds number $\mathrm{Re}_{\mathrm{t}}=\mathrm{u}^{\prime} \mathrm{l} / v_{\mathrm{u}}$ the normalised turbulent rootmean-square $(\mathrm{rms})$ velocity fluctuation $\mathrm{u}^{\prime} / \mathrm{S}_{\mathrm{L}}$, the ratio of $\mathrm{U}_{\mathrm{B}} / \mathrm{S}_{\mathrm{L}}$, integral length scale to thermal flame thickness ratio $1 / \delta_{\mathrm{th}}$, integral length scale to nozzle diameter ratio $\mathrm{l} / \mathrm{D}$, Damköhler number Da $=\mathrm{IS}_{\mathrm{L}} / \delta_{\mathrm{th}} \mathrm{u}^{\prime}$, and Karlovitz number $\mathrm{Ka}=\left(\mathrm{u}^{\prime} / \mathrm{S}_{\mathrm{L}}\right)^{3 / 2}\left(\mathrm{l} / \delta_{\mathrm{th}}\right)^{-1 / 2}$ are given in Table 1. Here $\mathrm{S}_{\mathrm{L}}$ is the unstrained laminar burning velocity, $\delta_{\text {th }}=\left(\mathrm{T}_{\mathrm{ad}}-\right.$ $\left.\mathrm{T}_{0}\right) / \max |\nabla \mathrm{T}|_{\mathrm{L}}$ is the thermal flame thickness with the adiabatic flame temperature $\mathrm{T}_{\mathrm{ad}}$ and the reactant temperature $T_{0}$. Note that the subscript ' $L$ ' refers to the unstrained laminar flame quantities. The heat release parameter $\tau=\left(\mathrm{T}_{\mathrm{ad}}-\mathrm{T}_{0}\right) / \mathrm{T}_{0}$ and the Zel'dovich number $\beta=$ $\mathrm{T}_{\mathrm{ac}}\left(\mathrm{T}_{\mathrm{ad}}-\mathrm{T}_{0}\right) / \mathrm{T}_{\mathrm{ad}}^{2}$ are taken to be 4.5 (except for case $\mathrm{T}$ where $\tau$ is reduced) and 6.0 respectively where $\mathrm{T}_{\mathrm{ac}}$ is the activation temperature. Standard values of Prandtl number $(\mathrm{Pr}=$ 0.7) and ratio of specific heats $\left(\gamma_{\mathrm{g}}=1.4\right)$ have been used. All non-dimensional numbers mentioned before have to be understood as inlet values here and in the remainder of the text.

Table 1: Characteristic properties of the different Bunsen flames.

\begin{tabular}{|c|c|c|c|c|c|c|c|c|c|c|}
\hline Case & $\mathrm{p}$ & $\mathrm{Re}$ & $\mathrm{Re}_{\mathrm{t}}$ & $\mathrm{U}_{\mathrm{b}} / \mathrm{S}_{\mathrm{L}}$ & $\mathrm{u}^{\prime} / \mathrm{S}_{\mathrm{L}}$ & $\mathrm{l} / \delta_{\mathrm{th}}$ & $\mathrm{l} / \mathrm{D}$ & $\mathrm{Ka}$ & $\mathrm{Da}$ & $\tau$ \\
\hline $\mathrm{A}$ & 1 bar & 399 & 13.30 & 6 & 1 & 5.20 & 0.2 & 1.94 & 5.00 & 4.5 \\
\hline $\mathrm{B}$ & 5 bar & 892 & 29.26 & 6 & 1 & 11.40 & 0.2 & 1.27 & 11.36 & 4.5 \\
\hline $\mathrm{C}$ & 10 bar & 1262 & 41.22 & 6 & 1 & 16.13 & 0.2 & 1.06 & 16.13 & 4.5 \\
\hline $\mathrm{D}$ & 1 bar & 399 & 41.22 & 6 & 3.1 & 5.20 & 0.2 & 10.59 & 1.61 & 4.5 \\
\hline $\mathrm{E}$ & 1 bar & 399 & 41.22 & 6 & 0.98 & 16.13 & 0.6 & 1.06 & 16.14 & 4.5 \\
\hline $\mathrm{H}$ & 10 bar & 2523 & 41.22 & 12 & 4 & 4.033 & 0.05 & 16.94 & 1.01 & 4.5 \\
\hline $\mathrm{I}$ & 10 bar & 391 & 41.22 & 6 & 1 & 16.13 & 0.645 & 1.06 & 16.13 & 4.5 \\
\hline $\mathrm{J}$ & 10 bar & 833 & 41.22 & 6 & 1 & 16.13 & 0.303 & 1.06 & 16.13 & 4.5 \\
\hline $\mathrm{K}$ & 10 bar & 1262 & 82.45 & 6 & 2 & 16.13 & 0.2 & 2.99 & 8.06 & 4.5 \\
\hline $\mathrm{L}$ & 10 bar & 1262 & 164.89 & 6 & 4 & 16.13 & 0.2 & 8.47 & 4.03 & 4.5 \\
\hline $\mathrm{M}$ & 10 bar & 1262 & 82.45 & 6 & 1 & 32.26 & 0.4 & 0.75 & 32.26 & 4.5 \\
\hline $\mathrm{O}$ & 10 bar & 1262 & 164.89 & 6 & 1 & 64.52 & 0.8 & 0.53 & 64.52 & 4.5 \\
\hline $\mathrm{R}$ & 1 bar & 1197 & 13.30 & 18 & 1 & 5.20 & 0.2 & 1.94 & 5.00 & 4.5 \\
\hline $\mathrm{T}$ & 1 bar & 399 & 13.30 & 6 & 1 & 5.20 & 0.2 & 1.94 & 5.00 & 2.0 \\
\hline $\mathrm{V}$ & 10 bar & 2523 & 41.22 & 12 & 8 & 4.033 & 0.025 & 67.76 & 0.25 & 4.5 \\
\hline
\end{tabular}


Inflow data reproducing the required turbulence properties has been generated using a modified version of the method suggested by Klein et al. [18] where the Gaussian filter in axial direction has been replaced by an autoregressive AR1 process in order to avoid excessive filter length in this direction caused by the small time step in the compressible flow solver. The reacting flow field is initialised by an unstrained premixed laminar flame solution with the geometry of a semi-sphere located at the inflow. Spatial derivatives for all internal grid points are evaluated using $10^{\text {th }}$ order central differences but the order of discretisation gradually drops to a one-sided $2^{\text {nd }}$ order scheme at the non-periodic boundaries. Time integration is carried out using an explicit 3rd order low storage Runge-Kutta scheme. All boundary conditions except the turbulent subsonic inflow are taken to be partially non-reflecting. Interested readers are referred to Refs. [19-22] for more details regarding the compressible flow solver SENGA.

The high pressure Bunsen flames have been simulated by adjusting viscosity and the Arrhenius parameters in such a way that $\mathrm{S}_{\mathrm{L}} \sim \mathrm{p}^{-0.5}, v_{\mathrm{u}} \sim \mathrm{p}^{-1}$ similar to the behaviour of methane-air flames. As a result of this, the Zel'dovich flame thickness $\delta_{\mathrm{L}}$ scales as $\delta_{\mathrm{L}} \sim \mathrm{p}^{-0.5}$ and the resolution has to be adjusted accordingly.

It has been demonstrated in the past that flame propagation statistics obtained from detailed chemistry simulations $[23,24]$ can be well captured using simple chemistry $[25,26]$. Furthermore, models which have been proposed based on the analysis of simple chemistry DNS of turbulent premixed flames with simplified transport have the potential to be valid even in the presence of detailed chemistry and transport (subject to minor adjustments) [27,28]. More references for the qualitative similarity of displacement speed and scalar gradient statistics between simple and detailed chemistry DNS results are provided [29]. Although the above discussion suggests that the assumption of a single step global reaction rate does not affect the 
qualitative nature of the results presented in this work future analyses with detailed chemistry and transport will be necessary for validation of the current findings.

The simulation domain is kept constant and corresponds for the 1 bar [5 bar] (10 bar) flame to a cube of $50 \delta_{\text {th }} \times 50 \delta_{\text {th }} \times 50 \delta_{\text {th }}$ [112 $\left.\delta_{\text {th }} \times 112 \delta_{\text {th }} \times 112 \delta_{\text {th }}\right]\left(159 \delta_{\text {th }} \times 159 \delta_{\text {th }} \times\right.$ $159 \delta_{\text {th }}$ ) which is discretised using a uniform Cartesian grid of $250 \times 250 \times 250$ [560×560×560] $(795 \times 795 \times 795)$ points. The nozzle diameter corresponds roughly to half the domain length. The simulation time when first statistics are taken is chosen to be larger than the maximum of two flow through and two eddy turnover times.

\section{RESULTS AND DISCUSSION}

It has been mentioned earlier that the focus of this work is to analyse curvature statistics and its relation to hydro-dynamic instability. The $\mathrm{c}=0.8$ isosurface of reaction progress variable is used as the flame surface throughout this work as the maximum value of reaction rate of progress variable takes place close to $\mathrm{c}=0.8$. It is important to understand that even a laminar Bunsen flame has not only an inherent negative flame curvature but also a negative skewness, when statistics are globally evaluated. This can be understood by considering a laminar Bunsen flame as illustrated in Fig. 3 together with the corresponding curvature PDFs. The blue line in Fig. 3 (b) corresponds to the simulation results. The curvature PDF model (red line) is based on a truncated cone which does not include the flame tip (which is assumed to begin once the flame cone reaches a diameter of $8 \delta_{\text {th }}$ ). Fig. 3(b) shows that this model closely represents the simulation results. By analyzing the curvature PDF model of a laminar Bunsen flame it is found that the skewness, or more precisely the curvature PDF, does not depend on the flame length. The PDF becomes stronger negatively skewed if the base (tip) diameter of the cone is increased 
(decreased). At the same time the variance changes with the variation of the base (tip) diameter of the cone.
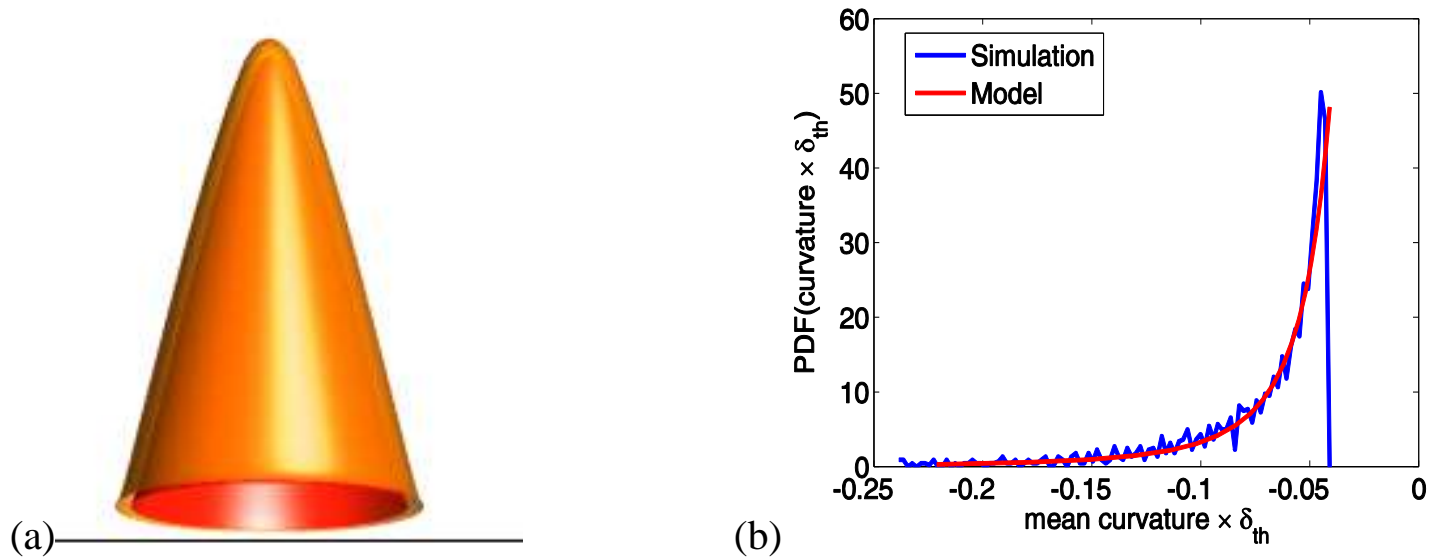

Figure 3. (a) Laminar Bunsen flame and (b) corresponding curvature PDFs. Mean curvature is normalised with the thermal flame thickness

Because the underlying mean flame geometry will influence curvature statistics of the turbulent Bunsen flame, it is essential to subtract the mean flame curvature (which depends on axial position) from local curvature values. This approach will be followed in the rest of the paper, where mean flame curvature is evaluated by averaging curvature values for each flow realization in $\mathrm{y}-\mathrm{z}$ planes, where mean flow is taken to align with the $x$-coordinate. In comparison to the curvature extracted from a time averaged mean contour of the DNS data this approach has the advantage that it can deal with instantaneous flame contours that might be broken or longer than the average flame length.

Turbulent Bunsen flames are considered next and Fig. 4 shows instantaneous views of reaction progress variable $\mathbf{c}$ isosurfaces for cases A, B and C. It can be seen from Fig. 4 that a characteristic cusp like wrinkling develops with increasing pressure (i.e. going from case A to B to C). The increasingly high negative values of curvature are reflected in the curvature PDFs 
shown in Fig. 4 (b). It becomes also apparent that the skewness of the curvatures PDFs increases with increasing pressure.

(a)

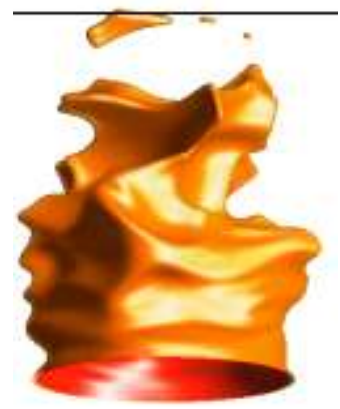

Case C

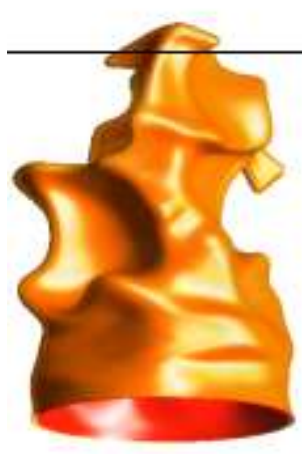

Case B

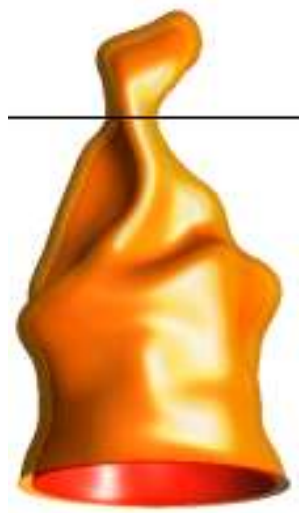

Case A (b)

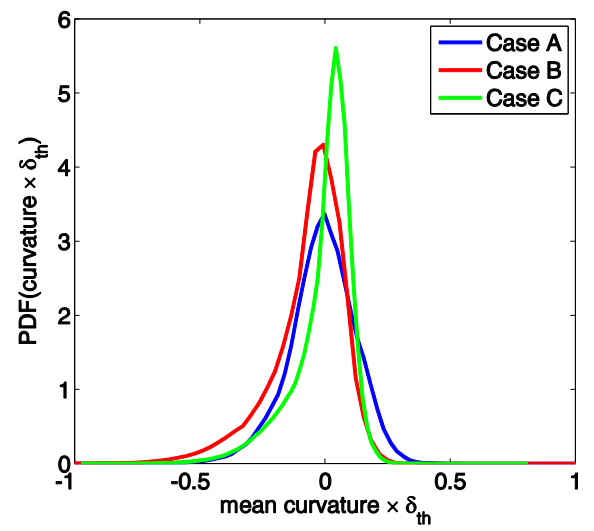

Figure 4. (a) Instantaneous view of $c$ isosurfaces for cases $C, B, A$. The value of $c$ increases from 0.1 (red) to 0.9 (yellow). The same color code will be used in the remaining figures. Cases C, B, A represent a 10 bar, 5 bar and 1 bar Bunsen flame. (b) Curvature PDFs for cases A-C. Mean curvature is normalised with the thermal flame thickness of the corresponding flame.

It is important to note that the curvature PDF in Fig. 4 (b) is normalized with the thermal flame thickness which scales as $\delta_{\mathrm{th}} \sim \mathrm{p}^{-0.5}$. This shows that the PDF distributions broaden with increasing pressure with higher probabilities of larger curvatures, indicating the enhancement of smaller scale flame front wrinkling which is consistent with the observations in Lachaux et al. [30] and Fragner et al. [31].

Second and third order moments of the curvature PDFs for different parameter variations are shown in Fig. 5, where it is clearly seen (Case C-B-A) that the PDF shows increased negative skewness with increasing pressure. 
(a)

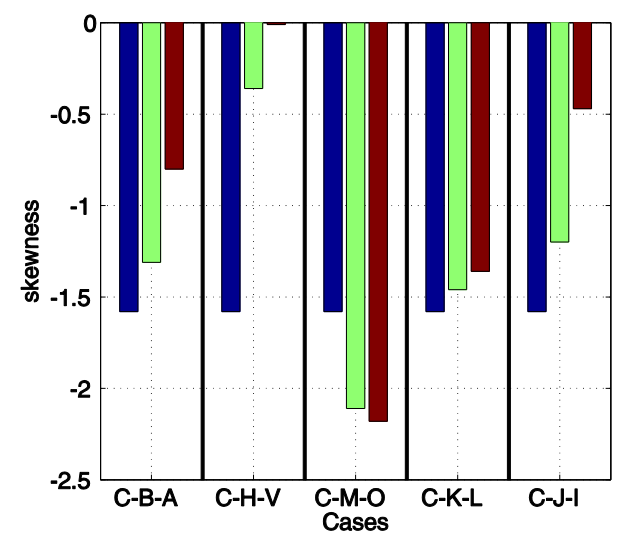

(b)

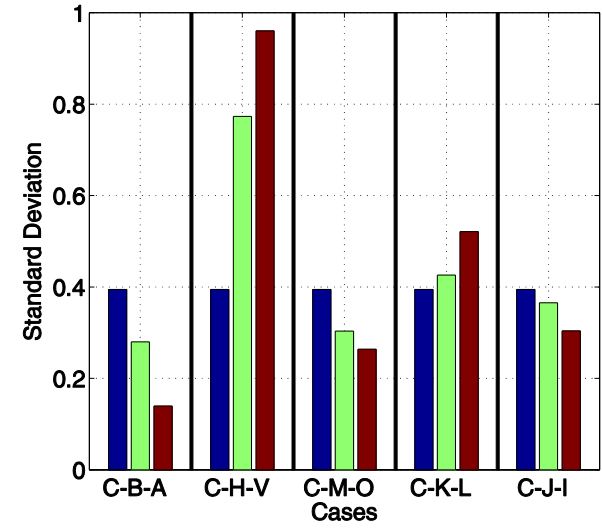

Figure 5. Third and second order moments of mean curvature normalized with $\delta_{t h}$. Skewness is shown in subfigure (a) and the standard deviation in subfigure (b)

It can be seen from Table 1 that $\mathrm{u}^{\prime} / \mathrm{S}_{\mathrm{L}}$ has been kept constant for cases $\mathrm{A}-\mathrm{C}$ and the same holds true for the integral length scale l of the inflow turbulence. It has been noted that $S_{L} \sim p^{-0.5}, v \sim$ $\mathrm{p}^{-1}$ and as a results of this the turbulent Reynolds number scales as $\operatorname{Re}_{\mathrm{t}} \sim \mathrm{p}^{0.5}$. The first question to answer is, if the increased skewness is a consequence of increasing the turbulent Reynolds number. Figure 6 compares three 1 bar flames, where in case D (case E) the turbulent velocity fluctuation (the integral length scale) have been increased to match the turbulent Reynolds number of the 10 bar case C. It is evident that the flame wrinkling increases (decreases) for Case D (Case E) but increased cusp formation is not visible.

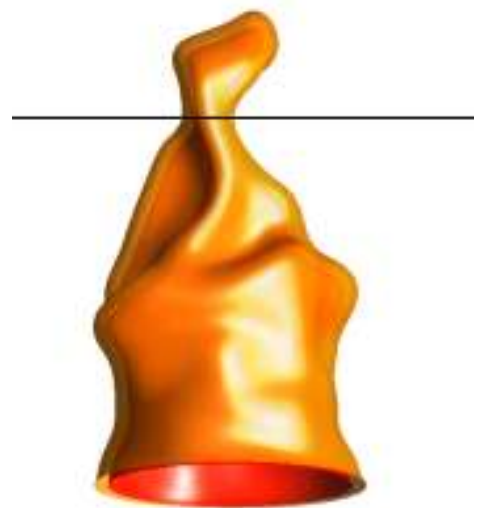

Case A

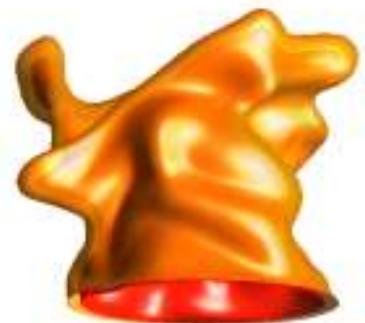

Case D

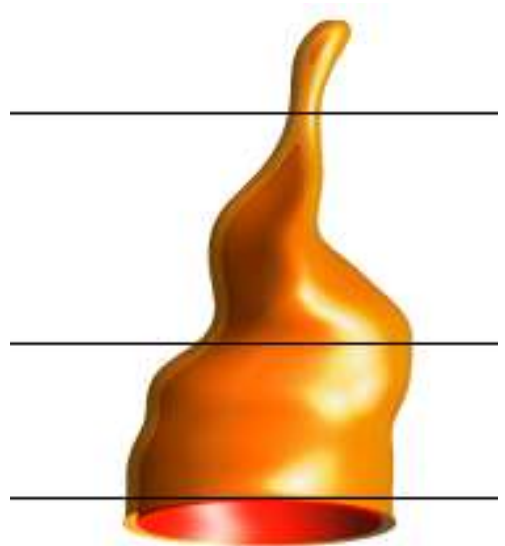

Case E

Figure 6. Three different 1 bar Bunsen flames where $u^{\prime} / S_{L},(l)$ has been increased for case D (case E) to match the turbulent Reynolds number of 10 bar Case $\mathrm{C}$. 
In order to understand if the negative skewness possibly originates from a hydro-dynamic DL instability it is important to calculate the critical wavelength which has been discussed earlier. A variety of theoretical models is available in the literature (see Ref. [11] for an overview). Table 2 reports the critical wavelength according to the relation by Matalon and Matkowsky [9] and according to the modified theory by Creta and Matalon [8], taking curvature and hydrodynamic strain rates into account. The critical wavelength differ roughly by a factor of two and this general behaviour is consistent with the observations in [8]. It is worth mentioning that Denet and Haldenwang [17] compare numerical results with theoretical predictions of Pelce and Clavin [10] and generally find that the theoretical predictions yield a smaller unstable range, especially towards larger wavenumbers.

Table 2: Critical wave length normalized with Zel'dovich flame thickness (nozzle diameter) for three different pressure levels according to $[8,9]$.

\begin{tabular}{|c|c|c|c|}
\hline$\lambda_{c} / \delta_{L}\left(\lambda_{c} / D\right)$ & 1bar & 5 bar & $10 \mathrm{bar}$ \\
\hline Creta \& Matalon [8] & $44.82(0.96)$ & $19.61(0.42)$ & $14.01(0.30)$ \\
\hline Matalon \& Matkowsky [9] & $23.11(0.50)$ & $10.50(0.23)$ & $7.00(0.15)$ \\
\hline
\end{tabular}

It becomes clear from Table 2 that some uncertainty remains regarding the determination of $\lambda_{c}$. Nevertheless, Table 2 suggests that $\lambda_{c}$ is clearly smaller than the nozzle diameter in the elevated pressure flames, whereas it is close to D (according to [8]) for the 1 bar case. For a DL instability to occur, a disturbance of wavelength $\lambda>\lambda_{c}$ is required. Creta et al. [7] consider the instability criterion $\mathrm{D}>\lambda>\lambda_{\mathrm{c}}$ for a Bunsen flame. Akkerman and Bychkov [5] argued that the characteristic length scale of the flow is determined by the distance from the orifice to the flame tip, which is larger than the orifice diameter. Finally, it is also possible to consider the circumference as the maximum length where a flame front instability could establish.

Figure 7 shows three different 10 bar Bunsen flames with identical inflow turbulence but decreasing nozzle diameter. The 10 bar case has been chosen for the diameter variation in order 
to allow for a large enough separation between flame thickness, critical wavelength and nozzle diameter. Visual inspection of the flame surface suggests that cusp formation is reduced for smaller nozzle diameters and this is confirmed from the skewness values reported in Fig. 5 (case C-J-I). Table 2 suggests that the nozzle diameter D/3 for Case I is equal or slightly larger than the critical wavelength. A few strongly negative curved surface elements are still visible but skewness is reduced from -1.58 for case $\mathrm{C}$ to -0.47 for Case $\mathrm{I}$, which is in agreement with the observation that the unstable wavelength range $\lambda_{c}<\lambda<D$ is considerably smaller.

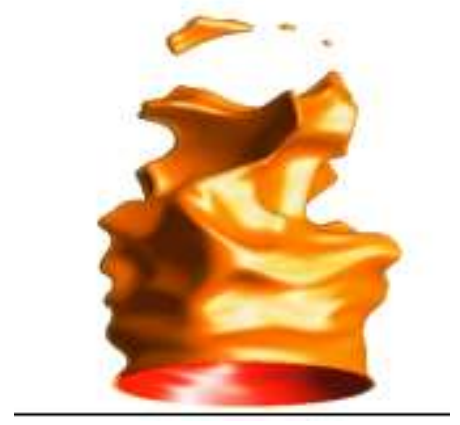

Case C

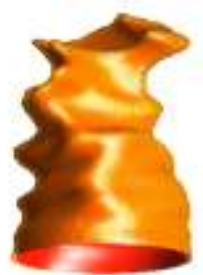

Case J

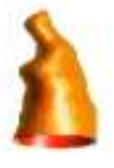

Case I

Figure 7. Three different 10 bar Bunsen flames with identical inflow turbulence but decreasing nozzle diameter: $D, 2 D / 3, D / 3$.

The influence of burner diameter on the turbulent burning velocity of premixed turbulent methane/air flames was analysed by Tamadonfar et al. [32]. Consistent with the present findings, it has been reported that increasing the burner diameter by a factor of 2 resulted in an increase of the turbulent burning velocity and this has been attributed to increased flame wrinkling. This result has been explained by the larger residence time for the burner with the larger nozzle diameter. An increase in the separation between the hydrodynamic length scale and the critical DL instability wavelength might, in the light of the present findings, be an alternative explanation. 
In order to better understand the factors influencing the turbulent flame surface case R (see Fig. 8) represents a variation of case A with an inlet velocity increased by a factor of three. According to Akkerman and Bychkov [5] the characteristic length scale of the flow is determined by the distance from the orifice to the flame tip and hence case $\mathrm{R}$ should have a larger unstable wavelength range resulting in increased flame instability. However, case $\mathrm{R}$ shows no tendency for increased cusp formation.

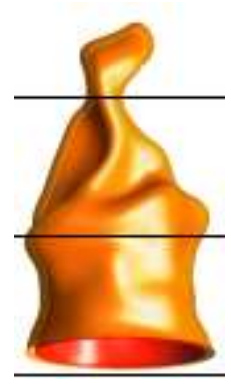

Case A

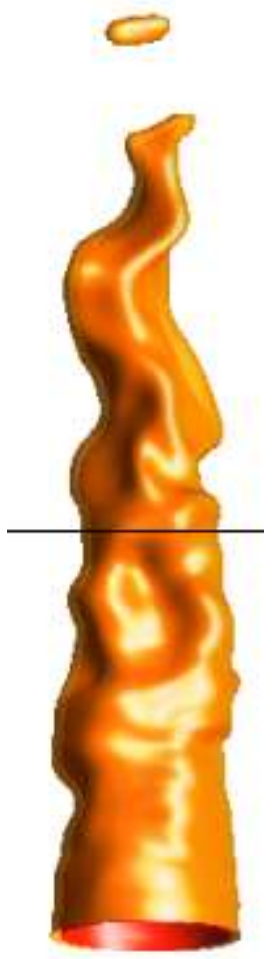

Case R

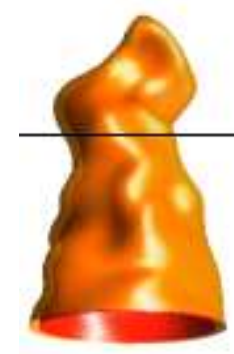

Case T

Figure 8. Three different 1 bar Bunsen flames. Case R corresponds to Case A with the difference that the inflow velocity is increased in order to obtain a longer flame. Case $\mathrm{T}$ has lower heat release parameter: $\tau=2$ whereas $\tau=4.5$ is used for the baseline. Note that all images are slightly scaled down due to space limitations.

The hydro-dynamic instability can only take place in the presence of heat release. Reducing the heat release parameter gives rise to decreased growth rates (see Fig. 1b). Therefore, it would be expected that hydro-dynamic instability is less pronounced for decreased heat release. In fact, the skewness for case T shown in Fig. $8(\tau=2)$ is about 0.2 larger (less negative) compared to 
case A. Decreasing heat release parameter further ( $\tau=1$, not shown) did not show an additional effect. It has to be mentioned that the analysis is complicated by the fact that the turbulence parameters change downstream of the inlet and the local turbulence levels at the flame front are affected by flame length (because of axial turbulence decay) and by heat release parameter (because of changing kinematic viscosity on the burned gas side and in the preheat zone).

The previous discussion was related to the question of onset of DL instability. Next, we concentrate on how turbulence influences flame geometry in the presence of DL instability and to this end only 10 bar flames are considered. Figures 9-11 show the effects of increasing the turbulent integral length scale (Cases C-M-O), increasing solely the turbulent velocity fluctuation $\mathrm{u}^{\prime} / \mathrm{S}_{\mathrm{L}}($ Cases $\mathrm{C}-\mathrm{K}-\mathrm{L})$, and increasing $\mathrm{u}^{\prime} / \mathrm{S}_{\mathrm{L}}$ but at the same time keeping $\mathrm{u}^{\prime} \times \mathrm{l}$ (and hence turbulent diffusivity respectively turbulent Reynolds number for the same pressure level) constant (Cases C-H-V).

Troiani et al. [16] and Akkerman and Bychkov [5] argued that an instability can develop if the ratio of the characteristic eddy frequency divided by linear growth rate $\sigma$ is smaller than unity, i.e.

$$
\beta=\frac{\omega_{t}}{\sigma}=\frac{2 \pi}{\tau_{\text {turb }} \sigma}=\frac{2 \pi \mathrm{u}^{\prime}}{\mathrm{l} \sigma}<1 ; \quad \tau_{\text {turb }}=1 / \mathrm{u}^{\prime}
$$

The underlying assumption of eq. 1 is the existence of a disturbance which subsequently grows as a result of hydro-dynamic instability. The time for this process is certainly limited by the characteristic eddy turnover time.

It is clear from Fig. 9 that increasing the turbulent integral scale lis effective in promoting negative skewness, whereas increasing $\mathrm{u}^{\prime} / \mathrm{S}_{\mathrm{L}}$ (see Fig. 10) results in a very small change of kurtosis (see Fig. 5). The latter fact is in line with the experimental observations reported in [6,7,16] and Bradley et al. [33]. When the turbulence level increases, the DL mechanism becomes relatively weaker and is finally overshadowed by turbulence. The most effective measure to suppress the instability is a combination of increasing $\mathrm{u}^{\prime} / \mathrm{S}_{\mathrm{L}}$ and decreasing $\mathrm{l}$ which 
ultimatively gives rise to a flame shape with zero skewness (see Fig. 11 and Fig 5). All observations regarding the variation of turbulence intensity, turbulent length scale and flame thickness are in line with the modified regime diagram presented by Chaudhuri et al. [34].

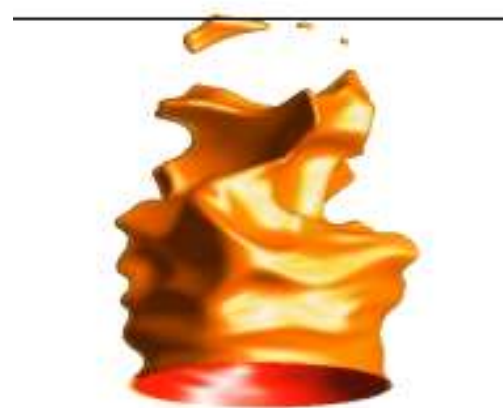

Case C

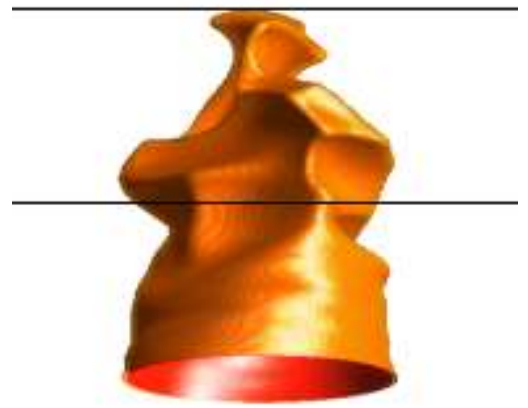

Case M

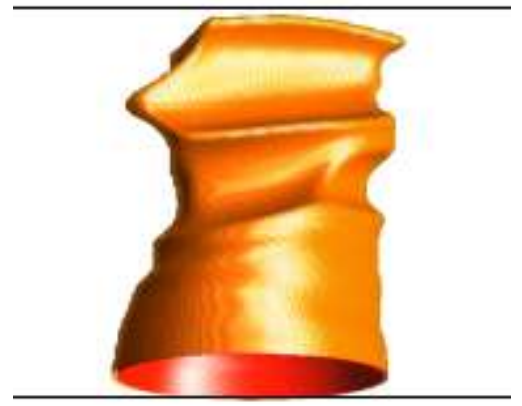

Case $\mathrm{O}$

Figure 9. Three different 10 bar Bunsen flames: $l$ increases from $1 / 5 D$ to $2 / 5 D$ to $4 / 5 D$ from case $\mathrm{C}$ to $\mathrm{M}$ to $\mathrm{O}$ whereas $u^{\prime} / S_{L}$ is kept constant.

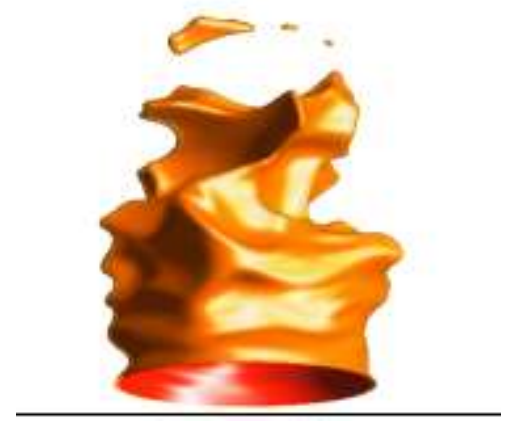

Case C

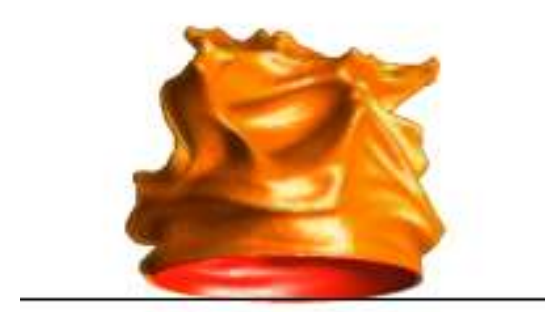

Case K

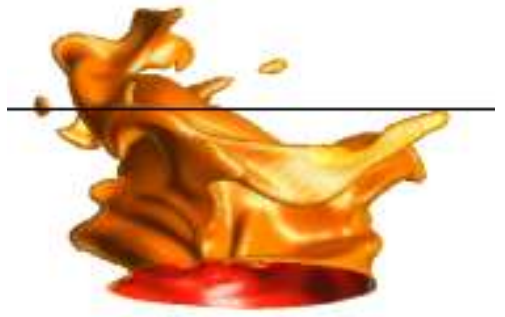

Case L

Figure 10. Three different 10 bar Bunsen flames: $u^{\prime} / S_{L}$ increases from 1 to 2 to 4 from case $\mathrm{C}$ to $\mathrm{K}$ to $\mathrm{L}$ whereas $l$ is kept constant.

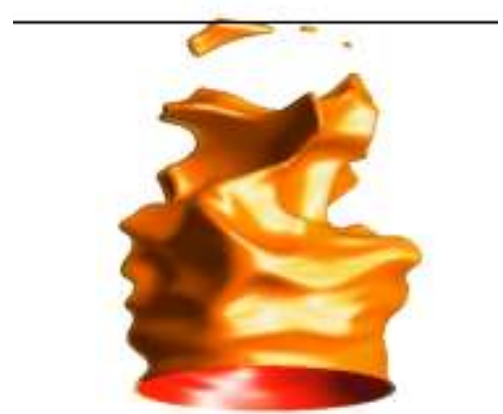

Case C

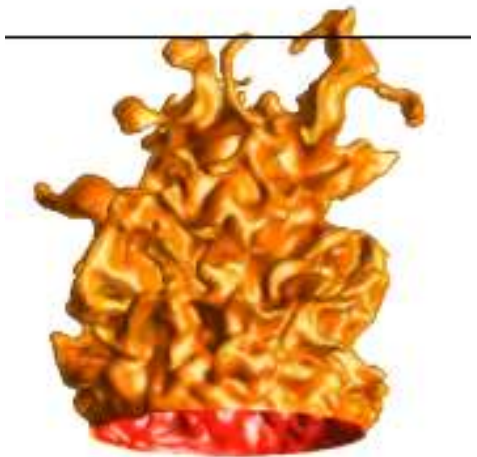

Case $\mathrm{H}$

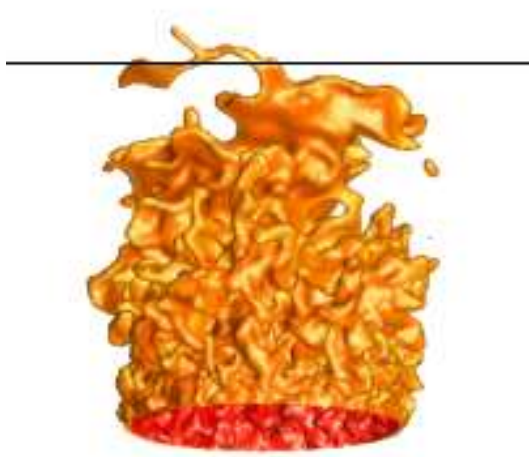

Case V

Figure 11. Three different 10 bar Bunsen flames where $u^{\prime} / S_{L}$, has been increased by a factor of four (eight) for case $\mathrm{H}$ (case $\mathrm{V}$ ) and the integral scale is reduced in order to keep the turbulent Reynolds number constant and equal to Case C. 
Equation 1 expresses that DL instability can compete with turbulence for $\beta<1$. However, it is to be noted that skewed curvature PDFs can occur even in the absence of hydro-dynamic instability. Following [14] it is suggested that the cusp formation time in the absence of DL instability [14] is proportional to the minimum of the inverse of local flame curvature (being negative as a requirement for cusp formation) and the inverse of flame speed. Assuming that the flame curvature of the initial disturbance that will give rise to cusp formation solely based on Huygen's principle, can be scaled as $\kappa \sim \mathrm{l}^{-1}$ with a constant of proportionality close to unity, the cusp formation time is $\tau_{\text {cusp }} \approx \mathrm{l} / \mathrm{S}_{\mathrm{L}}$. Assuming once more that the time for cusp formation is limited by $\tau_{\text {turb }}$ and introducing the characteristic time for the DL instability $\tau_{D L}=1 / \sigma$ the following criterion is obtained for pronounced cusp formation based on two alternative mechanisms:

$$
\min \left\{\tau_{\text {cusp }}, \tau_{D L}\right\}<\tau_{\text {turb }} \Leftrightarrow \min \left\{\frac{u^{\prime}}{S_{L}}, \frac{u^{\prime}}{l \sigma}\right\}<1
$$

In fact, negative skewness values in the DL stable region are reported [16], which can to some extent be explained by Eq. 2 .

It is important to note, that upon cusp formation the laminar flame speed is modified relative to its unstretched counterpart which is discussed in [8]. Due to the velocity component tangential to the flame front, flame elements move downstream towards the flame tip. As turbulence decays downstream of the nozzle exit, different turbulence conditions act on different flame elements as well as on the same flame element at different instants in time. Hence for simplicity no model constants are introduced in Eq. 2 which should be considered as a qualitative criterion. 
It is finally worth mentioning that besides skewness, Fig 5 (b) also shows the standard deviation of the curvature PDF. Results indicate that essentially an increase in turbulence intensity and a decrease in turbulent length scale are responsible for broadening the curvature PDF.

\section{MODELLING IMPLICATIONS}

According to Bray et al. [35] a model for the closure of mean turbulent reaction rate can be written as $\rho_{0} S_{L}^{0} I_{0} \Sigma$ where $\Sigma$ is the flame surface density (FSD) and $I_{0}$ is a correction factor accounting for the effects of strain and curvature on the laminar flame speed. The FSD $\Sigma$ can be obtained by algebraic closure models or by solving a suitable balance equation, whereas $I_{0}$ could be obtained from a laminar, full chemistry flame calculation that can be stored in a database. However, such a parametrization requires that strain rate and curvature are well characterized [36]. It is known that the average of strain rate in a turbulent flame has a finite positive value [36] which acts to reduce the laminar flame speed. If the flame response to curvature is considered to be linear and curvature has a zero mean then $I_{0}$ could solely be modelled from a knowledge of the distribution of the strain rates. For Bunsen flames the mean curvature is negative. This means that the flame is negatively stretched which acts to increase the laminar burning velocity and also the displacement speed. Exemplarily it is mentioned that the volume integrated reaction rate for case $\mathrm{A}$ is about $8 \%$ larger than $\rho_{0} S_{L}^{0} \Sigma$. The validity of the relation $S_{T} / S_{L}=A_{T} / A_{0}$, where $A_{0}$ is the area perpendicular to the direction of the flame propagation, is discussed in [38]. It is unlikely that the linear flame speed stretch relation holds true for highly skewed curvature PDFs with large curvature values. In this regard, explicit modelling of mean curvature contribution might be necessary and even modelling the unresolved curvature [37] might play a role in the context of LES of hydro-dynamically unstable flames. Finally, it is mentioned that DL instability gives rise to negative skewness and 
increased flame area. Explicit modelling of this effect might be required especially for high pressure flames with low turbulence intensity, where DL instability is likely to occur.

\section{CONCLUSIONS}

A three-dimensional DNS database of Bunsen flames representing ambient and elevated pressure environment has been established using a simplified chemistry assumption in order to carry out an extensive parametric study. The surface geometry has been analyzed in detail and it is found that the high pressure flames exhibit large negative curvature values giving rise to pronounced negative skewness of the curvature PDF. The observed flame shapes are consistent with experimental observations [1] and it is confirmed that the DL instability promotes this phenomenon [7]. It is also found that the criteria for determining the range of unstable wavelength are bound by the critical wavelength and the nozzle diameter, which are consistent with the observations made in [7] for two-dimensional Bunsen flame simulations. The strength of the instability is strongly dependent on the incoming turbulence in particular the integral length scale and the turbulence intensity. It is also found that negative curvature PDF skewness can be observed for flames where DL instability is either weak or entirely absent and a criterion for the occurrence of negative skewness of curvature PDF has been suggested. For the flames under investigation the negative skewness could be observed for relatively large turbulence intensities. However, for values of $\mathrm{u}^{\prime} / \mathrm{S}_{\mathrm{L}}$ at the order of 10 combined with a relatively small turbulent scale a symmetric curvature PDF was observed. Finally modelling implications of the aforementioned observations have been discussed. Future work will be needed to extend this analysis to turbulent flame speed correlations and to the explicit modelling of resolved and unresolved curvature distributions in the context of LES. 


\section{ACKNOWLEDGEMENTS}

Computer resources for this project have been provided by the Gauss Centre for Supercomputing / Leibniz Supercomputing Centre under grant: pr74ra. NC is grateful to ARCHER for computational support.

\section{COMPLIANCE WITH ETHICAL STANDARDS}

Computer resources for this project have been provided by the Gauss Centre for Supercomputing / Leibniz Supercomputing Centre under grant: pr74ra. NC is grateful to ARCHER for computational support.

\section{CONFLICT OF INTEREST}

The authors declare that they have no conflict of interest. 


\section{REFERENCES}

[1] Kobayashi H., Experimental study of high-pressure turbulent premixed flames, Experimental Thermal and Fluid Sciences 26, 375-387, (2002).

[2] Keppeler R., Tangermann E., Allaudin A. and M. Pfitzner: LES of Low to High Turbulent Combustion in an Elevated Pressure Environment. Flow, Turbulence and Combustion, 92(3): 767-802, (2014).

[3] Keppeler R. and Pfitzner M.: Modelling of Landau-Darrieus and thermo-diffusive instability effects for CFD simulations of laminar and turbulent premixed combustion. Combustion Theory and Modelling (2014), http://dx.doi.org/10.1080/ 13647830.2014.975747.

[4] Creta F. and Matalon M., Propagation of wrinkled turbulent flames in the context of hydrodynamic theory, J. Fluid Mech., 680, 225-264, (2011).

[5] Akkerman V. and Bychkov V. Velocity of weakly turbulent flames of finite thickness, Combustion Theory and Modelling, 9, 323-351, (2005).

[6] Fogla N., Creta F. and Matalon M., The turbulent flame speed for low-to-moderate turbulence intensities: Hydrodynamic theory vs. experiments, Combustion and Flame, 175, 155-169, (2017).

[7] Creta F., Lamioni R., Lapenna P.E. and Troiani G., Interplay of Darrieus-Landau instability and weak turbulence in premixed flame propagation Physical Review E 94, 053102, (2016).

[8] Creta F. and Matalon M., Strain rate effects on the nonlinear development of hydrodynamically unstable flames, Proceedings of the Combustion Institute, 33, 1087-1094, (2011).

[9] Matalon M. and Matkowsky B.J., Flames as gasdynamic discontinuities, J. Fluid Mech., 124, 239-259, (1982).

[10] Pelce P. and Clavin P. Influence of hydrodynamics and diffusion upon the stability limits of laminar premixed flames, J . Fluid Mech., 124, 219-237, (1982). 
[11] Lipatnikov A.N., and Chomiak J., Molecular transport effects on turbulent flame propagation and structure, Progress in Energy and Combustion Science 31, 1-73, (2004).

[12] Shepherd I.G., Ashurst W.T., Flame front geometry in premixed turbulent flames, Proc. Combust. Inst., 24, 485-491, (1992).

[13] Peters, N. “Turbulent Combustion”, Cambridge University Press, UK (2000).

[14] Law C.K. and Sung C.J., Structure, aerodynamics, and geometry of premixed flamelets, Progress in Energy and Combustion Science, 26, 459-505, (2000).

[15] Plessing T., Kortschik C., Mansour M.S., Peters N., and Cheng R.K., Measurement of the turbulent burning velocity and the structure of premixed flames on a low swirl burner, Proc. Combust. Inst. 28, 359-368, (2000).

[16] Troiani G., Creta F. and Matalon M., Experimental investigation of Darrieus-Landau instability effects on turbulent premixed flames, Proceedings of the Combustion Institute, $35,1451-1459,(2015)$.

[17] Denet B., Haldenwang P., A Numerical Study of Premixed Flames Darrieus-Landau Instability, Combust. Sci. and Tech., 104, 143-167, (1995).

[18] Klein M., Sadiki A., Janicka J.: J. Comp. Physics, 186, 652-665, (2003).

[19] Jenkins, K.W. and Cant R.S., "Direct numerical simulation of turbulent flame kernel", In Recent Advances in DNS and LES, Springer, pp 191-202 (1999).

[20] Klein M., Chakraborty N., Jenkins K., and Cant R.S., Effects of initial radius on the propagation of premixed flame kernels in a turbulent environment Physics of Fluids 18, 055102, (2006).

[21] Chakraborty, N., Klein M. and Cant R.S., Effects of turbulent Reynolds number on the displacement speed statistics in the thin reaction zones regime of turbulent premixed combustion, Journal of Combustion, Article number 473679, (2011). 
[22] Chakraborty N., Klein M., Swaminathan N., Effects of Lewis number on the reactive scalar gradient alignment with local strain rate in turbulent premixed flames. Proceedings of the Combustion Institute, 32, (2009), 1409-1417.

[23] T. Echekki, J.H. Chen, Unsteady strain rate and curvature effects in turbulent premixed methane-air flames, Combust. Flame, 106, 184-202 (1996).

[24] N. Peters, P. Terhoeven, J.H. Chen, T. Echekki, Statistics of flame displacement speeds from computations of 2-D unsteady methane-air flames, Proc. Combust. Inst., 27, 833-839 (1998).

[25] N. Chakraborty, S. Cant, Unsteady effects of strain rate and curvature on turbulent premixed flames in an inflow-outflow configuration, Combust. Flame, 137:129-147 (2004)

[26] N. Chakraborty, M. Klein, R.S. Cant, Stretch rate effects on displacement speed in turbulent premixed flame kernels in the thin reaction zones regime, Proc. Combust. Inst., $31,1385-1392(2007)$.

[27] J. Lai, M. Klein and N. Chakraborty, Direct Numerical Simulation of Head-On Quenching of Statistically Planar Turbulent Premixed Methane-Air Flames Using a Detailed Chemical Mechanism, Flow Turb. Combust., https://doi.org/10.1007/s10494$\underline{018-9907-5}(2018)$.

[28] M. Klein, C. Kasten, N. Chakraborty, N. Mukhadiyev and H.G. Im, Turbulent Scalar Fluxes in Hydrogen-Air Premixed Flames at Low and High Karlovitz Numbers, Combust. Theor. Modell, accepted (2018).

[29] M. Klein, D. Alwazzan and N. Chakraborty, A direct numerical simulation analysis of pressure variation in turbulent premixed Bunsen burner flames-Part 1: Scalar gradient and strain rate statistics, Computers and Fluids, https://doi.org/10.1016/j.compfluid.2018.03.010 (2018). 
[30] T. Lachaux, F. Halter, C. Chauveaua, I. Gökalp and I.G. Shepherd, Flame front analysis of high-pressure turbulent lean premixed methane-air flames, Proc. Combust. Inst., 30, 819-826 (2005).

[31] R. Fragner, F. Halter, N. Mazellier, C. Chauveau and I. Gökalp, Investigation of pressure effects on the small scale wrinkling of turbulent premixed Bunsen flames, Proc. Combust. Inst. 35, 1527-1535 (2015).

[32] P. Tamadonfar and Ö.L. Gülder, Effect of burner diameter on the burning velocity of premixed turbulent flames stabilized on Bunsen-type burners, Experimental Thermal and Fluid Science 73, 42-48 (2016).

[33] D. Bradley, M. Lawes , K. Liu , M.S. Mansour , Measurements and correlations of turbulent burning velocities over wide ranges of fuels and elevated pressures, Proc. Combust. Inst. 34, 1519-1526 (2013).

[34] Chaudhuri, V.Y. Akkerman, C.K. Law, Spectral formulation of turbulent flame speed with consideration of hydrodynamic instability, Phys. Rev. E 84, 026322 (2011).

[35] Bray, K.N.C., Libby, P.A., Moss, J.B. "Unified modelling approach for premixed turbulent combustion - Part I: General Formulation”, Comb. Flame, 61:87-102 (1985).

[36] Cant R.S., Rutland C.J., Trouve A, Statistics for laminar flamelet modeling, Stanford Univ., Proceedings of the 1990 Summer Program, (1990).

[37] Chakraborty N. and Cant R.S., Direct Numerical Simulation analysis of the Flame Surface Density transport equation in the context of Large Eddy Simulation, Proceedings of the Combustion Institute, 32, 1445-1453, (2009).

[38] Gülder Ö.L. Contribution of small scale turbulence to burning velocity of flamelets in the thin reaction zone regime, Proceedings of the Combustion Institute, 31, 1369-1375, (2007). 